\title{
Evaluating Community Engagement through Argumentation Maps: A Public Participation GIS Case Study
}

\section{Claus Rinner}

Ryerson University

\section{Michelle Bird}

Ryerson University

\section{Please Cite:}

Rinner, C., \& Bird, M. (2009). Evaluating community engagement through argumentation maps: A public participation GIS case study. Environment and Planning B: Planning and Design, 36(4), 588-601.

doi: $10.1068 / \mathrm{b} 34084$

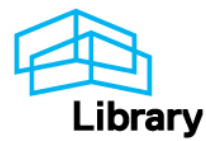


[final draft post-refereeing for institutional repository - not suitable for citations]

\title{
Evaluating Community Engagement through Argumentation Maps - A Public Participation GIS Case Study
}

\author{
Claus Rinner, Michelle Bird \\ Department of Geography \\ Ryerson University \\ 350 Victoria Street \\ Toronto ON M5B2K3 \\ Canada \\ crinner at ryerson.ca
}

\begin{abstract}
Significant advances in Public Participation Geographic Information Systems technology and online mapping platforms have not translated into enhanced citizen participation in democratic planning processes. This study contributes to addressing this gap by evaluating the engagement of members of an urban community in sustainable neighbourhood planning through argumentation mapping. The study provided an online, public discussion forum together with a neighbourhood map to which the participants could link their discussion contributions. Based on participation statistics, contents of contributions, and responses to a survey we discuss the participants' technical and engagement experiences. The sixteen registered participants lived within or near the "Queen West Triangle” in downtown Toronto, Canada. They rated themselves as experienced computer users and consequently found the participation in the online discussion forum to be easy. The contributions showed a great degree of interest and knowledge in the issues of sustainable community development. However, while the majority of participants also rated themselves as comfortable with map reading, they found the handling of the online neighbourhood map difficult and did not use the option to link their comments to the map.
\end{abstract}




\section{Introduction}

The desire to build sustainable and inclusive societies has come with the recognition of the value of involving the public in planning and decision-making processes. It is believed that incorporating local knowledge and opinions into decision-making creates results that are more readily accepted and relevant within communities (Kingston et al, 2000). The study of Public Participation Geographic Information Systems (PPGIS) deals with the confluence of social activity participatory activities, grassroots organizations, governmental decision-making, planning and policy-making - and Geographic Information Systems (GIS) (Niles and Hanson 2001). PPGIS concepts and technologies are evolving in many directions including approaches to mapping human argumentation processes (Rinner 2006).

The argumentation map model provides a framework for distributed, asynchronous discussions that relate to space (Rinner 2001, 2005). The model includes explicit links between argumentation elements and geographic objects which enables participants in planning debates to reference the places (e.g. buildings, streets, parks) they are discussing in their contributions. Subsequently, those existing geographic references allow for a map-based access of the debate. The "ArguMap" prototype used in this case study was developed by Keßler (2004). This implementation of the argumentation map concept has been evaluated in terms of its usability (Sidlar and Rinner 2007) and the utility of the underlying concepts (Sidlar and Rinner, in press) but the present case study is its first use in a realistic context with public access.

The case study was conducted in collaboration with Active 18, a "citizens, residents and businessowners association of Ward 18” (Active 18, 2007) in west-central downtown Toronto, Canada. The association has formed as a result of concerns over proposed high-density residential development 
within the "Queen West Triangle”. The goal of Active 18 is to raise awareness regarding the potential effects of the development plans on the community. The organization is interested in promoting holistic, sustainable development in the area. To date, Active 18 has organized a community charrette which produced a community vision and plan for the neighbourhood. The association welcomed the proposal of using the ArguMap tool to increase dialogue and engagement in the issue.

Thus, this paper describes a reality-based case study using the ArguMap prototype in order to evaluate its role as a PPGIS and collaborative spatial decision support tool. In particular, we attempt to gain a greater understanding of two elements of using the prototype: the users' experience with the tool as a technological application, and the potential benefits of using the argumentation map concept to support community engagement in urban planning.

This paper first discusses the role of the World-Wide Web to support spatial decision-making, and more specifically, geographically referenced discussions. Methods for evaluating the usefulness of PPGIS applications are also presented. Next, we describe the data preparation, setup, participants, and procedure of the ArguMap case study on sustainable community development. The results of the case study are summarized with respect to participation statistics, contents of contributions, and responses to a user survey. Finally, we discuss the technical usability of the ArguMap prototype as well as the community engagement achieved through the use of argumentation mapping, and we provide an outlook on future work in this area. 


\section{Public Participation GIS and Argumentation Mapping}

\subsection{The Role of the World-Wide Web}

The application of Web-based GIS (WebGIS) to community decision-making processes offers a range of potential societal benefits. Firstly, these tools respond to the criticism that GIS technology is elitist by offering solutions that are accessible to non-experts (Craig 1998; Carver and Peckham 1999). Online tools also offer an alternative to traditional public meetings in that they do not require in-person attendance. By operating on the Internet, access to these GIS is not restricted by time or location (Kingston et al, 2000; Carver 2001; Peng 2001; Dragicevic and Balram 2004). In addition, individuals uncomfortable with speaking in public can voice their opinions in a more detached environment, and a wider, more representative audience can be reached (Craig 1998; Carver and Peckham 1999; Kingston et al, 2000).

WebGIS applications hold advantages for planners as well. The nature of the input received from the public in this format is generally more complex but also easier to process and analyze than data collected from traditional public meetings (Carver and Peckham 1999; Kingston et al, 2000; Carver 2001). For example, in a traditional public meeting a note-taker would have to record vocalized comments from participants. Conversely, contributions made via a WebGIS will be in electronic format, and thereby easier to organize and analyze. In addition, online contributions can take on forms other than text contributions, such as polls. WebGIS also hold benefits for community organizations by allowing them to analyze, explore and present information in a multifaceted and meaningful manner (Craig 1998).

It is important to note the challenges faced by Web-based participatory GIS applications. The most significant barrier given that these tools have been developed for public participation, is that of the 
“digital divide”. Varying degrees of access to technology and computer literacy are issues that all WebGIS applications encounter (Carver and Peckham 1999; Kingston et al, 2000; Carver 2001; Peng 2001).

As part of the scientific discourse of PPGIS, the technology-driven perspective on WebGIS was accompanied by occasional discussions on the conceptual-theoretical level of how GIS applications interact with political processes and power structures in society (e.g. Pickles 1995). More recently, Craig et al, (2002, p.4) discuss “differential access to geographic information and technology”, the “representation of multiple realities ... within GIS”, the "identification of the potential beneficiaries of participatory GIS projects”, and in general, the inclusiveness of GIS-driven decision-making processes in local communities. Ramasubramanian and Quinn (2004) point to the transparency of the participatory process and the accountability of the organizers as some of the critical aspects of PPGIS implementations. With reference to the increasing use of GIS technology by non-profit organizations and citizen groups, Sieber (2004) argues for a second generation of GIS that would incorporate technical mechanisms to represent multiple, possibly conflicting, perspectives on places and integrate local knowledge.

\subsection{Decision Support vs. Discussion Support}

Using an approach proposed by Stern and Fineberg (1996) for public participation in environmental decision-making, Jankowski and Nyerges (2003) acknowledge the two facets of collaborative spatial decision-making processes: the analytic and the deliberative element. "The analytic component provides technical information that ensures broad-based, competent perspectives. [...] The deliberative component provides an opportunity to give voice to choices about values, alternatives and recommendations” (Nyerges 2005). On this level, the analytic components could be associated 
with common decision support tools such as multi-criteria decision analysis while the deliberative component could be matched with discussion support tools such as online forums.

More specifically, Jankowski and Nyerges (2003) outline the three steps of the analytic-deliberative process as

- identification of issues,

- measurement of possible decision outcomes, and

- $\quad$ discourse among stakeholders.

These steps bear some resemblance with the three phases of Simon's (1976) model of decision making phases - intelligence, design, and choice - where the deliberative step provides for a formal acknowledgement of the role of citizen participation in the choice phase.

This approach can be compared with a distinction between different types of spatial decision support systems (SDSS). Keenan (1997) positions standard GIS software as data-driven SDSS that can be used for the exploration of issues, while the common definition of SDSS (Densham 1991) assumes model-driven systems that serve to integrate data and simulate decision outcomes. To these two types of SDSS, a “discourse-based SDSS” could be added as a third type to represent tools that support annotation of decision alternatives and discussion among stakeholders. Table 1 summarizes these parallels that may help to clarify the terminologies used by different research communities. 
Table 1: The parallels between Simon's (1976) model of decision-making, the analytic-deliberative approach (Stern and Fineberg 1996), computer-supported tasks in decision-making, and types of SDSS

\begin{tabular}{|l|l|l|l|}
\hline Decision-Making & Analytic-Deliberative & Computer-Supported & SDSS Types \\
Phases & Approach & Tasks & \\
\hline intelligence & identification of issues & data exploration & data-driven \\
\hline design & measurement of possible & data integration and & model-driven \\
& decision outcomes & simulation & \\
\hline choice & discourse among stakeholders & $\begin{array}{l}\text { annotation and } \\
\text { discussion }\end{array}$ & $\begin{array}{l}\text { discourse- } \\
\text { driven? }\end{array}$ \\
\hline
\end{tabular}

Drawing from argumentation theory, computer-supported cooperative work and PPGIS concepts, Rinner (2001) developed the argumentation map model to represent computer-based, spatiallyrelated discussions. This model allows argumentation elements (i.e. comments) to refer to geographic objects and visa versa. Argumentation elements can also refer to graphic reference objects that the user can create on a map (to represent subjectively defined areas). With the same goal of supporting spatial “understanding”, Horita (2000) presents an approach for argumentative representation in policy discourse.

Among the earliest and most comprehensively studied PPGIS implementations that allowed for map annotations was Carver et al.’s (2001) Web application that was based on the GeoTools Java mapping toolkit, HTML forms, and PERL scripts for server-side processing and storage of user input. Similarly, Voss et al. (2004) combine Dito, an online groupware application for structured discourse, with CommonGIS, a Java-based interactive mapping tool. Rinner’s (2001) argumentation map model was implemented in a software prototype called “ArguMap” by Keßler (2004). This 
Web-based discussion support tool uses a combination of the MySQL database and Java Servlets on the server-side, and the mapping package GeoTools Lite and a custom discussion forum on the client-side running in the participant's Web browser. The design goals for this prototype included user-friendliness and the use of geo-spatial interoperability standards (Keßler et al, 2005). Other map-based discussion support tools that have been developed recently include GeoDF (Tang and Coleman 2005, Tang 2006) and MapChat (Leahy et al, 2006).

\subsection{Evaluation of Public Participation GIS}

While the technology exists for WebGIS to be deployed in a variety of contexts, there is a lack of real-world applications (Carver 2001; Carver et al, 2001; Steinmann et al, 2004). A number of barriers can account for this shortage. The majority of applications are developed and implemented by academic researchers. Due to limited funding and multiple responsibilities, it is difficult for these individuals to maintain applications over extended periods of time (Steinmann et al, 2004). There is also a disconnect between WebGIS developers and planners resulting in a lack of awareness of the technical possibilities in the planning profession. Additionally, planners tend to be reluctant to give up decision-making power for fear of trivialization of the planning process, and/or reservations towards the ability of the public to give valid opinions (Carver and Peckham 1999; Carver et al, 2001; Steinmann et al, 2004). Haklay (2003) notes technical challenges with respect to the representation of local knowledge and making visualizations and spatial queries understandable to an untrained audience. Lastly, due to the nature of the Internet there is the issue of bias in system authoring and control, copyright, and confidentiality (Carver and Peckham 1999).

Selected applications of WebGIS have allowed for the development of evaluation processes. Studies by Peng (2001) and Carver et al. (2001) outline recommendations for a successful application and 
assessment modes. These include allowing the user to explore, experiment, and evaluate geographic data, with the capacity to build various scenarios and participate in some type of forum; providing data that are understandable and non-partisan; and creating a transparent process that allows the user to submit information to those with final decision-making power, and to see the results in order to build trust. Comparative research related to evaluating WebGIS has been conducted by Steinmann et al. (2004) in a study that examined the interactivity, usability, and visualization functions of twelve American and European applications. On the conceptual level of PPGIS as a scientific endeavour, Sieber (2006) notes the lack of measures of PPGIS effectiveness and proposes a framework for understanding PPGIS. Similar factors are used by Jankowski and Nyerges (2003) to outline a method for empirical assessment of PPGIS use.

Case studies in the particular area of map-based discussion support are scarce and often conducted by researchers without involvement of external participants. An early public participation case study with map-based discussion support was conducted by Carver et al. (2001). Voss et al. (2004) describe the evolution of their participatory tool using experiences from three case studies that were conducted in 2002 and 2003. Zhao and Coleman (2006) report the ongoing implementation of a pilot project for the GeoDF tool while Keßler’s (2004) ArguMap prototype has been evaluated in terms of its usability by Sidlar and Rinner (2007). The utility of the underlying argumentation map concept was assessed by Sidlar and Rinner (in press). Both of these case studies involved student participants while the present case study is the first use of the tool in a realistic context with public access. 


\section{Methodology of the Case Study}

\subsection{Data Preparation and Application Setup}

The geographical scope of this case study was bound by the "Queen West Triangle”, an area identified and labelled by the Active 18 citizens association. The Queen West Triangle is bound by Queen Street West to the north, Dovercourt Road to the east, and the Canadian National rail corridor to the southwest.

This project used Keßler’s (2004) ArguMap prototype in order to evaluate its role as a spatial decision support tool in the context of community engagement. The tool consisting of a Java client and Java servlets was set up on top of an Apache Web server with a tomcat servlet engine and a MySQL database. ArcGIS was used to create shapefiles of the study area that were integrated into the ArguMap application. The map layers included a portion of an orthophoto from the City of Toronto as well as labelled street lines and areal building footprints. Figure 1 is a screenshot of the application.

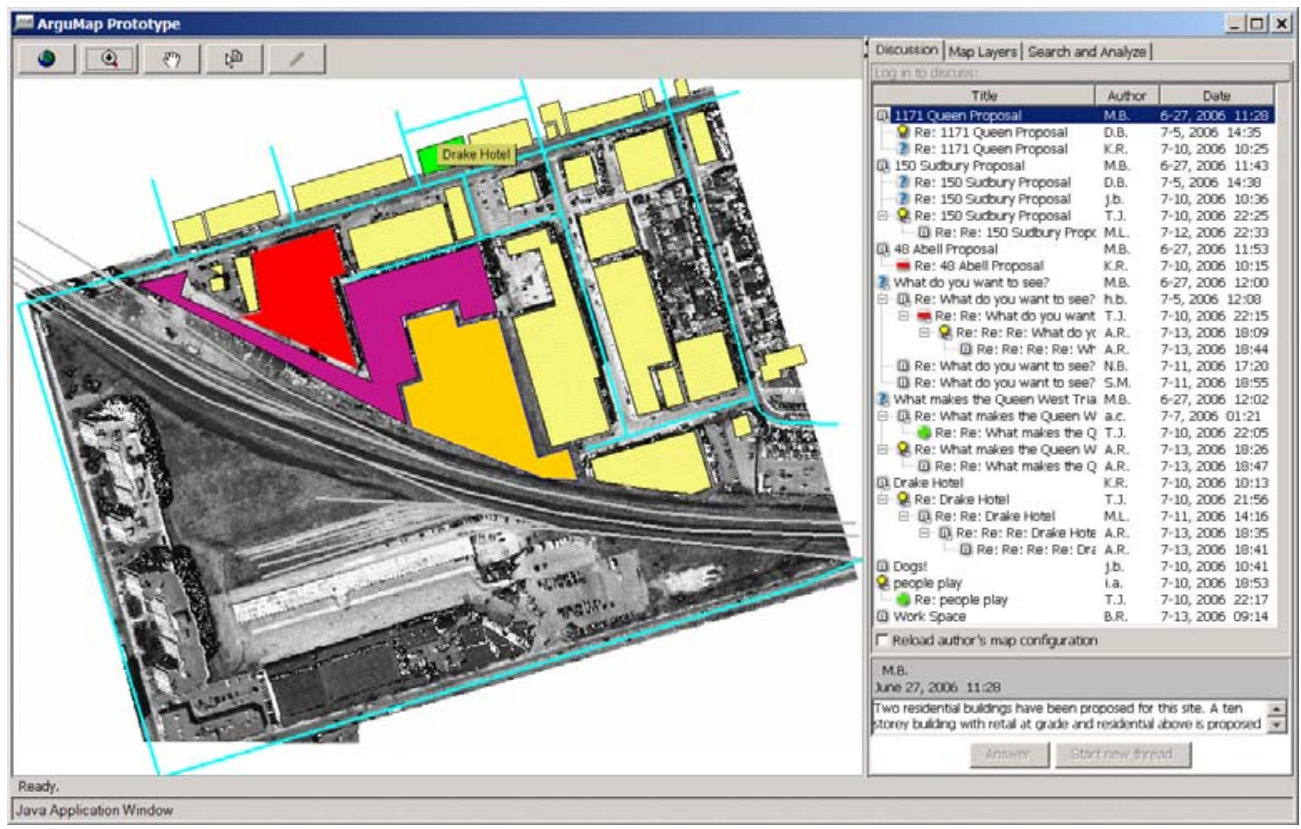

Figure 1: Screenshot of the ArguMap application for the Queen West Triangle 


\subsection{Participants and Procedure}

An introductory Web page was developed to instruct users on the background of the study and on how to use the ArguMap. Information about four steps in operating the ArguMap was given: launching the application, creating a user account, reading the discussion, and joining the discussion. Screenshots were used to highlight the functionality of the tool. From this instruction page, the user could launch the application.

The ArguMap went live on Tuesday, June 27, 2006, and operated for 18 days until Friday, July 14, 2006. A link to the application on the Active 18 homepage was activated. Thus, the case study was open to the general public. However, in collaboration with the Active 18 association, the ArguMap application was specifically promoted to residents and business owners living and operating within the Queen West Triangle. A promotional flyer was designed and 200 copies were distributed to all households and businesses in the Queen West Triangle. The flyer was also sent to the Active 18 email list of approximately 100 recipients.

In order to facilitate the discussion, five starter messages were submitted to the discussion forum by the authors. The text of these contributions contained information about the current urban fabric in the Queen West Triangle and the proposed changes, and focused on stimulating the expression of opinions on these developments.

The discussion was closed on July 14 by disabling the login function of the application and removing the link from the Active 18 homepage. The participants were contacted via email requesting their feedback through an online survey instrument. 


\section{Results}

\subsection{Participation Statistics}

A total of 16 participants registered to and subsequently logged in to the ArguMap application. Of these 16 participants, 12 actively posted contributions. There were a total of 26 contributions made to the discussion, not including the starter messages. Three duplicate contributions from the same participant were removed before the analysis.

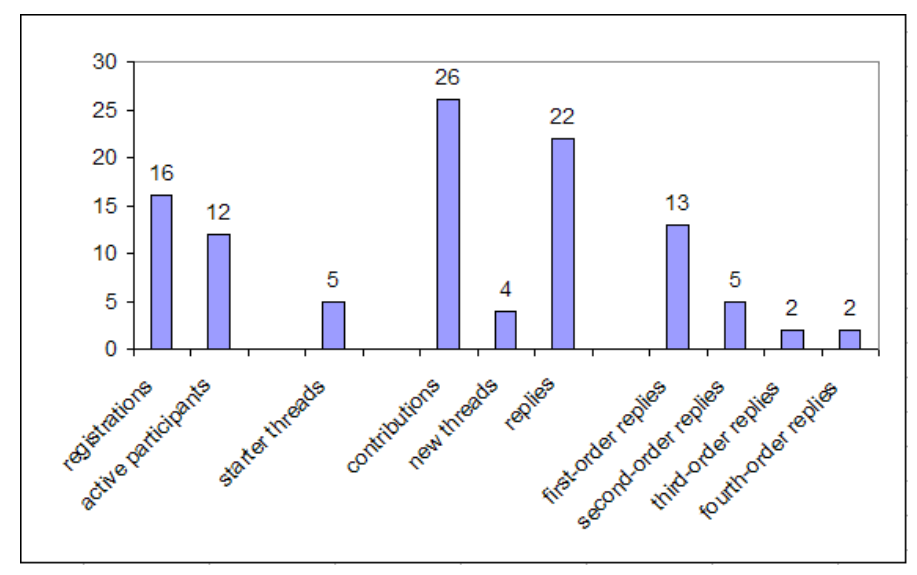

Figure 2: Participation statistics

With the exception on the starter messages, only one other contribution had a reference to the map.

Four new discussion threads were created. The average number of replies to a thread was 2.44 (median 3), with the total number of replies being 22. Of these 22 replies, 13 were $1^{\text {st }}$ order (direct replies), five were $2^{\text {nd }}$ order (reply to reply), two were $3^{\text {rd }}$ order (reply to $2^{\text {nd }}$ order reply), and two were $4^{\text {th }}$ order (reply to $3^{\text {rd }}$ order reply) (see Figure 2). The average number of contributions per participant was 1.63 (median 1.5). 


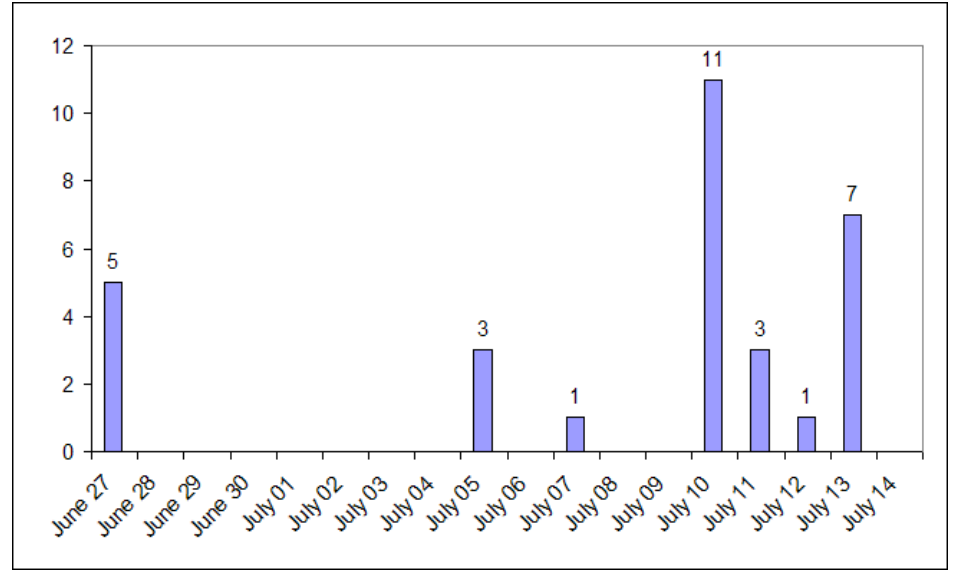

Figure 3: Time line of contributions

Examination of the dates when contributions were submitted showed that there was no activity during the first nine days of the study, and that all contributions were submitted during the later part of the study period (see Fig. 3).

\subsection{Contribution Content}

The ArguMap prototype offered a platform for a dynamic discussion of the issues in the Queen West Triangle. In total, the discussion contained nine threads including those based on the five starter messages. To provide an example, one of the starter messages is entitled "1171 Queen West Proposal" and reads "Two residential buildings have been proposed for this site. A ten storey building with retail at grade and residential above is proposed on Queen Street West, and a 26storey residential tower is proposed for the southern portion of the site." One of the replies to this thread reads "How would a 26-storey tower feel when you are walking around this area? I agree that we should be increasing density in general in Toronto to reduce urban sprawl, but I think it is also important to think about scale. Tall buildings can make an area lose its feeling of connectivity and intimacy....they block out the sun creating shadows.” 
While it is impossible to summarize the participants' opinions towards the development in the Queen West Triangle, it is possible to identify a number of key themes that reoccur in their messages. Table 2 illustrates these themes that are either implicitly or explicitly stated within the contributions. These themes are: supporting "smart” growth as championed by Active 18; promoting unique design ideas; the desire for public/green space, accessibility for all income groups, and maintaining the area's “culture”. Some contributions contained more than one theme.

Table 2: Recurring themes in contributions

\begin{tabular}{|l|l|}
\hline Theme & Number of occurrences \\
\hline Pro “smart” development & 6 \\
\hline Desire for green/public space & 6 \\
\hline Maintaining “culture” & 5 \\
\hline Accessibility for all income groups & 3 \\
\hline Desire for unique design & 3 \\
\hline
\end{tabular}

\subsection{Survey Results}

Once the Active 18 ArguMap discussion was closed, each of the participants received an email requesting their feedback via an online survey. $100 \%$ of the participants fully completed and returned the survey. The survey questionnaire was composed of five parts:

1. The Active 18 Issue,

2. Creating an Engaging Discussion,

3. Using the ArguMap Tool,

4. Demographics, and

5. Additional Feedback. 
Part 1 of the survey shows that 13 of the 16 participants have been to the Queen West Triangle, and that half of the respondents were aware of Active 18 and/or the proposed changes.

Part 2 of the survey included questions designed to assess the user's experience with respect to learning and expressing opinions about the development issue. Participants were asked to rate six statements on a scale from strongly agree to strongly disagree. Participants offered positive feedback with respect to becoming engaged in the Active 18 issue. Agree was the option most frequently selected for statements focusing on increased understanding of the issue, voicing opinions, and connectedness.

The questions contained in part 3 of the survey were designed to elicit responses regarding the ease of use of the application's functionality. Users were asked to rate their opinion with regards to certain functions by selecting one option: very easy, easy, somewhat difficult, difficult, or I did not use this function. The responses for this part of the survey are less uniform than the previous parts. Participants found it easier to use the discussion board functions of the tool and more difficult to use the GIS elements. For example, "reading the various discussion threads" was rated by $100 \%$ of users as very easy (18.75\%) or easy (81.25\%), but “zooming and moving around the map” was more challenging as $75 \%$ of participants replied this was somewhat difficult or difficult. The ease of use of overall participation was rated easy by $50 \%$ of the participants. $31.25 \%$ replied it was somewhat difficult, and $18.75 \%$ rated the experience difficult.

Questions referring to demographics composed part 4 of the survey. The case study included 9 female participants and 7 male participants. Most of the participants (56.25\%) fall into the 30-39 age 
range. Only $12.5 \%$ of participants actually live within the Queen West Triangle, but 50\% live within $5 \mathrm{~km}$ of the study area, and an additional $25 \%$ live within $10 \mathrm{~km}$. The remaining participants live within the Greater Toronto Area. With respect to their computer and geographic literacy, 100\% of the participants strongly agreed or agreed to the statement "I am comfortable with basic computer operating functions", while $87.5 \%$ of users strongly agreed or agreed that they "learn new things easily on computers”. Lastly, 75\% of participants strongly agreed that they "have a good sense of direction and understand maps easily”.

The last part of the survey offered a possibility for open-ended feedback. Five participants responded to this question and qualified the argumentation map as valuable, interesting, a worthwhile experience, and a good idea. However, three respondents reported problems with the map navigation functions while two had problems installing the Java applet.

\section{Discussion}

This PPGIS case study has provided a wealth of information that offers an excellent opportunity to understand the impacts of an argumentation mapping tool in a number of different ways. To explore these varied implications, we first provide a general critique discussing advantages and disadvantages of specific elements of the study’s research design. The second subsection discusses the users’ technical experience with the ArguMap prototype. The third subsection focuses on the users’ engagement experience, analyzing the utility of the argumentation map as a tool for public participation. 


\subsection{General Critique}

The case study results have both encouraging and disappointing aspects. The most positive element is the contents of the discussion generated through the application. The contributions contained excellent ideas and the beginnings of constructive dialogue between the participants. The contents of the contributions are actionable and could be incorporated into the Active 18 association's documentation.

However, the functionality forming the very basis for the ArguMap tool - the ability to link comments to places shown on a map - was used only once among all contributions. An explanation could be that the discussion related to the Queen West Triangle did not necessitate spatial referencing. The users tended to comment with expressions of general ideas for this relatively small area, not with specific suggestions for places within the area. This underlines the need to test the ArguMap for larger study areas with discussions that are more focused on particular places.

Furthermore, the number of participants was small as in other PPGIS case studies. 16 individuals registered and 12 contributed after a distribution of 200 flyers and a notice via the Active 18 email list of approximately 100 recipients. This could be a result of the timing of the case study. When the investigators contacted the neighbourhood association in early April 2006, the association had just completed a community charrette on March 5, 2006. This charrette was attended by the steering committee (a strong group of influential professionals) and led by an experienced architect and urban designer. Prominent urban planners, designers, architects, development economists, landscape architects, cultural producers and other members of the community attended this one-day event to develop a community vision for the Queen West Triangle. If the case study was operated in conjunction with this highly promoted event, the results might have been different. As it stands, the 
ArguMap application was launched after the charrette with the community vision document already published. Individuals involved in the charrette process may have found participating in the ArguMap discussion redundant. While Active 18 was supportive of the study, they did not champion the project because it somewhat lacked context after the charrette. This underscores the need to implement the PPGIS tool within the processes and needs of community organizations.

The number of participants was also a result of the recruitment method. If an in-person training session or information session could be organized, the number of participants and the nature of feedback received might be different. As well, participation required leaving a valid email address which may have deterrent some potential participants.

Finally, it should be noted that time was needed for the discussion to gain momentum. During the first week of the application's operation, there were no contributions. This identifies the need to allow time for a discussion to build, and the value of having starter messages to stimulate the discussion.

\subsection{Technical Experience}

The participants' technical experience can be divided into two phases: the initial start-up process (informed by the access statistics) and the participation process (informed by the participation statistics and the user survey).

The user experience to launch the application contained a number of barriers and challenges. A major challenge for many individuals was downloading Java. The user survey reported that half of the users found accessing the ArguMap somewhat difficult or difficult. This identifies the need to 
create a more streamlined, user-friendly process to ensure that the user does not become disengaged before they even launch the application.

In terms of the users’ participation process, although 16 users registered, only 12 contributed comments. This illustrates that people are interested in the issue (or the technology) but do not necessarily want to participate. From the discussion and the user survey, it can be seen that once users have launched the application, the participation process became easier to navigate. Overall, the users were more comfortable with the discussion board aspect of ArguMap, and not as comfortable with the GIS aspect of the tool. Functions of the ArguMap that are similar to other applications present on the Internet were highly utilized and mostly rated "easy" in the user survey. It was more challenging for participants to use the GIS functions of ArguMap such as understanding the map, panning and zooming, and turning map layers on and off. The fact that these basic GIS functions presented a challenge to some users implies that using more complex functionality (such as linking a contribution to an object on the map) would be difficult. In order for participants to use the spatial reference function, the tool-tips for the map and basic GIS functions need to be improved. Labeling the features of the map including the road names and buildings (instead of dynamic pop-up label) would facilitate the user's understanding of the map. This is especially true in the case of the Queen West Triangle where the irregular shapes of the proposal sites present an unfamiliar perspective of the area. As well, the icons for map manipulation functions could be more expressive, especially the one for the unfamiliar functionality of geographic referencing. These icons could be bigger, use text and have a more informative tool-tip. Furthermore, when a user starts a new thread or replies to a thread, the writing space could include a prompt message that motivated geographic referencing. In essence, further testing is required to understand how to assist users in optimizing the GIS functions of ArguMap. 
Thus, the user's technology experience indicates that while participants comprehend the discussion board within the tool, further development is needed to increase understanding of the GIS aspects of ArguMap. The results of the survey illustrate mixed response to its overall ease of use. This data demonstrates the great potential for improving the technical components of the tool.

\subsection{Engagement Experience}

The ArguMap application provided a valuable and relevant platform for disseminating information regarding developments in the Queen West Triangle and gathering opinions on this subject. The user's engagement experience can be understood by examining the nature of the contributions, inferences of the participant's characteristics, and results of the participant survey.

The comments contributed by the users in the ArguMap discussion were intelligent and insightful. They demonstrate that ArguMap has a good capability for gathering public opinions on decisions related to space. These ideas could be incorporated into formal planning processes or could be used by a community organization as a source of data. The tool provided a platform for users to voice their opinions and respond to others. This specific discussion generated strong ideas illustrated by the reoccurring themes discussed in section 4.2. However, the discussion needs to be placed within the context of the characteristics of the participants to attempt to understand the type of user the case study attracted, and the types of users excluded from the discussion.

The content of the contributions indicates that the users participating in the ArguMap discussion had a general understanding of urban issues and community development. Participants used informed language such as "gentrification”, “urban sprawl”, “density”, “sustainability”, “urban planning”, 
“map layers”, “spatial location”, and “official plan” illustrating their awareness of such concepts. The participant survey provides further insight into the nature of the users. The participants were comfortable with technology, as well as with maps. More than half the participants live in or within 5km of the Queen West Triangle, and were aware of the issue and/or Active 18 before the ArguMap experience. All of this information infers a study group that is computer literate, comfortable with technology, and has a high level of awareness regarding urban planning issues.

The user groups not included in the study can be inferred. From this information, neither teenagers nor retired individuals participated in the study. This is especially important in the Queen West Triangle situation as Active 18 has noted that the majority of houses located on Dovercourt Road south of Queen are owned by (mainly retired) individuals of Italian and Portuguese descent. When the researcher hand-distributed flyers in this area, this observation was confirmed. These homeowners were polite, but were not aware of the developments and were confused by the idea of using the Internet to voice their opinions. The fact that all of the participants were comfortable with basic computer operating functions also speaks to the exclusion of individuals without access or knowledge of computers. This is an important recognition, as it is these individuals (most likely from lower income groups without access or knowledge of using online applications) that are most affected by the process of gentrification. As interest in development increases and property values rise, it is the marginalized groups of the community that will be forced to move or change their lifestyles in response to the changes. It is important to note that accessibility is more complex than access to a computer and the Internet. Ramasubramanian (2000) discusses the need for a "critical world view". It is not enough for these individuals to be taught how to use a computer; it is necessary to expose them to the ideas that compose the issue and ways of vocalizing their thoughts through writing. Only then does the technology have meaning and relevance. 
In summary, the participants of this ArguMap case study represented a certain demographic that is computer literate and aware of urban processes. For a tool aiming at public participation, this highlights the lack of representation of the community and the challenges of accessibility. This inequality was mainly a result of the research design, dictated mainly by the restriction of time. Indeed, further studies could achieve a more representative milieu of opinions by operating initiatives such as a offering face-to-face workshops or getting more relevant community organizations involved.

The final element in understanding the user's engagement experience involves examining the participant survey. Overall, the application was very well received by the participants. This is important as a fundamental requirement of a public participation tool. There was a fairly good response to questions related to becoming more engaged in the issue itself once the case study experience was complete. More than half the users felt they had a greater understanding of the issue, that their interest in the issue had increased since using ArguMap, and agreed that they felt more connected to the issue. This demonstrates the ability of the technology to truly engage the public.

\section{Conclusions and Outlook}

This case study has afforded valuable insight into the challenges and benefits of using the ArguMap technology as a method of public participation. Like many other PPGIS applications, ArguMap offers the benefits of bringing GIS technology to those not familiar with the technology and offering a viable alternative to attending public planning meetings. As a Web-based application ArguMap is available at any time and can be accessed from any Web-enabled location, allowing participation from a wider range of participants. The quality of the contributions made in the study illustrated how 
ArguMap can generate ideas from the public that can be valuable in leveraging the position of community organizations.

The study has also underlined a number of challenges in using this technology. The main challenge is that of accessibility. The results illustrated that while users were comfortable with the discussion board aspects of ArguMap, they did not readily understand the GIS aspects of the tool. Therefore, there is a need to further research methods of making this functionality more user-friendly. If this “tech-savvy” user group found this challenging, how much of a barrier would it present to other user groups with less computer know-how? This represents a great opportunity for further developments.

This study has also illustrated the importance of context and process. While the study was enthusiastically received by Active 18, it occurred during an unfavourable time. This highlights the importance of using technology during the most advantageous context, and ensuring it is highly integrated into the community organization's process so that they play a strong role in championing the application and they find value in its results.

A number of future recommendations can be made with regards to ArguMap. The first recommendation focuses on implementing an improved version of the prototype in a case study situation that has a greater emphasis on specific spatial elements. A study of a site with specific proposals for a variety and number of objects (buildings, roads, etc.) within the map would necessitate linking contributions to places. In this manner, such a case study could provide a stronger quantitative analysis of the utility of the functions of the application, as well as gathering results that are more directed and actionable. Further research could examine how these results affected development outcomes as well. 
A second recommendation involves understanding how an urban planner or community organization would use the results of the ArguMap. What types of results are most valuable? How does the data get processed and analyzed? What additional functionality (such as a report generator) would benefit planners and community organizations? In this manner, the application could be developed further to benefit the intended user groups.

A final recommendation focuses on how to package the ArguMap tool so that it can be fully implemented from installation to study completion by targeted provider groups, such as urban planners and community groups. Currently ArguMap is a research prototype. What process would be needed so that non-programmers could install the application? What barriers does the provider encounter? Could an installation interface be designed to guide the upload process? At the writing of this paper, an improved version of the ArguMap using Google Maps is in development. This would reduce the amount of GIS knowledge needed to operate the technology. Research in this direction would move the technology towards true integration in relevant sectors.

As technology continues to advance, so does our ability to develop it into manifestations that benefit our societies. While challenges are ever present, so is the desire and ability to overcome these barriers and create valuable, relevant solutions. The Active 18 ArguMap is an illustration of this, and can only serve to inform improvements and solutions for future developments. 


\section{Acknowledgements}

We would like to thank the participants in the online discussion for their time and their thoughtful contributions to the forum. Michelle Gay of Active 18 was very helpful in designing the promotional flyer and setting up a link to the forum on the association's Web site. Carsten Keßler and Martin Rapp assisted with setting up the ArguMap application and preparing the map layers. Constructive suggestions by four anonymous reviewers are gratefully acknowledged. Jyothi Kumari provided additional support for preparing the final manuscript. Partial funding for this research was received form the Canadian GEOIDE Network of Centres of Excellence in Geomatics.

\section{References}

Active 18, 2007, Active 18 Association’s Web site. Available at http://www.active18.org (last accessed: 22 July 2007)

Carver S, Peckham R, 1999, “Using GIS on the Internet for Planning”, in Geographical Information and Planning Ed. J Stillwell (Springer, New York) pp 371-390

Carver S, 2001, “Public Participation Using Web-Based GIS” Environment and Planning B 28(6) 803-804

Carver S, Evans A, Kingston R, Turton I, 2001, “Public Participation, GIS and Cyberdemocracy: Evaluating Online Spatial Decision Support Systems” Environment and Planning B 28(6) $907-921$

Craig WJ, 1998, “The Internet Aids Community Participation in the Planning Process” Computers, Environment and Urban Systems 22(4) 393-404

Craig WJ, Harris TM, Weiner D, 2002, Community Participation and Geographic Information Systems (London: Taylor and Francis) 
Densham PJ, 1991, “Spatial decision support systems”, in Geographical Information Systems:

Principles and Applications Eds DJ Maguire, MF Goodchild, DW Rhind (Longman, London) pp 403-412

Dragicevic S, Balram S, 2004, “A Web GIS collaborative framework to structure and manage distributed planning processes” Journal of Geographical Systems 6(2) 133-153

Haklay M, 2003, “Usability Evaluation and PPGIS: Towards a User-Centred Design Approach” International Journal of Geographical Information Science 17(6) 577-592

Horita M, 2000, “Mapping policy discourse with CRANES: spatial understanding support systems as a medium for community conflict resolution” Environment and Planning B 27(6) 801-814

Jankowski P, Nyerges T, 2003, “Toward a Framework for Research on Geographic InformationSupported Participatory Decision-Making” URISA Journal 15(APA I) 9-17

Keenan P, 1997, “Using a GIS as a DSS Generator”, Working Paper MIS 95-9, University College Dublin

Keßler C, 2004, “Design and Implementation of Argumentation Maps” Diploma Thesis, Westfälische Wilhelms-Universität Münster, Germany. Available at http://www.carstenkessler.de/argumap/diploma.pdf (last accessed: 22 July 2007)

Keßler C, Rinner C, Raubal M, 2005, “An Argumentation Map Prototype to Support DecisionMaking in Spatial Planning”, in Proceedings of AGILE 2005 - 8th Conference on Geographic Information Science Eds F Toppen, M Painho, 26-28 May 2005, Estoril, Portugal, pp 135-142

Kingston R, Carver S, Evans A, Turton I, 2000, “Web-based Public Participation Geographical Information Systems: An Aid to Local Environmental Decision Making” Computers, Environment and Urban Systems 24(2) 109-125 
Laituri M, 2003, “The Issue of Access: An Assessment Guide for Evaluating Public Participation Geographic Information Science Case Studies” URISA Journal 15(2) 25-32

Leahy M, Hall GB, Findlay D, Nicholls T, Feick R, 2006, “Implementation of Synchronous, Spatially-Referenced Discussions Between Multiple Users with Open-Source Web GIS and Database Tools”, in Proceedings of FOSS4G2006 - Free and Open Source Software for Geoinformatics, 11-15 September 2006, Lausanne, Switzerland

Niles S, Hanson S, 2001, “A New Era of Accessibility: Or Is It?” Workshop on Access to Geographic Information and Participatory Approaches Using Geographic Information, Spoleto, Italy, 6-8 December 2001. Available at http://www.spatial.maine.edu/ onsrud/Spoleto/NilesHanson.pdf (last accessed 22 July 2007) Nyerges T, 2005, “Scaling-Up as a Grand Challenge for Public Participation GIS” Directions Magazine (online), dated 20 September 2005. Available at http://www.directionsmag.com/article.php?article_id=1965 (last accessed 22 July 2007). Peng Z, 2001, “Internet GIS for Public Participation” Environment and Planning B: Planning and Design 28(6) 889-905

Pickles J, 1995, “Representations in an Electronic Age”, in Ground Truth: The Social Implications of Geographic Information Systems Ed. J Pickles (Guilford Press, New York) pp 1-30 Ramasubramanian L, 2000, “Knowledge Production and Use in Community-based Organizations: Examining the Impacts and Influence of Information Technologies”, Doctoral Research Paper, University of Wisconsin-Milwaukee

Ramasubramanian L, Quinn A, 2004, “Participatory GIScience: Progress or Illusion”, paper presented at the URISA Public Participation GIS Conference, 18-20 July 2004, Madison, Wisconsin. Available at http://www.urisa.org/prev/PPGIS/2004/Prelim/absMonday.htm (last accessed 22 July 2007) 
Rinner C, 2001, “Argumentation Maps: GIS-based discussion support for on-line planning” Environment and Planning B: Planning and Design 28(6) 847-863

Rinner C, 2005, “Computer Support for Discussions in Spatial Planning”, in GIS for Sustainable Development Ed. M Campagna (CRC Press, Boca Raton, FL) pp 167-180

Rinner C, 2006, “Argumentation Mapping in Collaborative Spatial Decision Making”, in Collaborative GIS Eds Dragicevic S, Balram S (Idea Group, Hershey, PA) pp 85-102

Sarjakoski T, 1998, “Networked GIS for Public Participation - Emphasis on Utilizing Image Data” Computers, Environment and Urban Systems 22(4) 381-392

Sidlar C, Rinner C, 2007, “Analyzing the Usability of an Argumentation Map as a Participatory Spatial Decision Support Tool” URISA Journal 19(1) 47-55

Sidlar C, Rinner C, in press, “Utility Assessment of a Map-Based Online Geo-Collaboration Tool” Journal of Environmental Management (to appear in early 2008)

Sieber RE, 2004, “Rewiring for a GIS/2” Cartographica 39(1) 25-39

Sieber RE, 2006, “Public Participation Geographic Information Systems: A Literature Review and Framework” Annals of the Association of American Geographers 96(3) 491-507

Steinmann R, Krek A, Blaschke T, 2004, “Analysis of Online Public Participatory GIS Applications with respect to the Differences Between the US and Europe”, in Proceedings of the 24th Urban Data Management Symposium, 27-29 October 2004, Chioggia, Italy

Stern PC, Fineberg V (Eds), 1996, Understanding Risk: Informing Decisions in a Democratic Society, Report by National Research Council, Committee on Risk Characterization (National Academy Press, Washington, DC)

Tang T, Coleman DJ, 2005, “Design of a GIS-based Online Discussion Forum for Participatory Community Planning”, in Proceedings of the 98th Annual Conference of the Canadian Institute of Geomatics, 13-15 June 2005, Ottawa, Canada 
Tang MY, 2006, Design and Implementation of a GIS-Enabled Online Discussion Forum for Participatory Planning M.Sc.E. Thesis, Department of Geodesy and Geomatics Engineering. Technical Report No. 244, University of New Brunswick, Fredericton, New Brunswick, Canada, $151 \mathrm{pp}$

Voss A, Denisovich I, Gatalsky P, Gavouchidis K, Klotz A, Roeder S, Voss H, 2004, “Evolution of a participatory GIS” Computers, Environment and Urban Systems 28(6) 635-651

Zhao J, Coleman DJ, 2006, “GeoDF: Towards a SDI-based PPGIS application for E-Governance”, in Proceedings of the GSDI-9 Conference, 6-10 November 2006, Santiago, Chile 